\title{
Migrations climatiques : quel rôle pour le droit international?
}

Climate migration: which role for international law?

Michèle Morel et Nicole de Moor

\section{(2) OpenEdition \\ 12 Journals}

\section{Édition électronique}

URL : http://journals.openedition.org/conflits/18580

DOI : $10.4000 /$ conflits. 18580

ISSN : $1777-5345$

Éditeur :

CCLS - Centre d'études sur les conflits lilberté et sécurité, L'Harmattan

\section{Édition imprimée}

Date de publication : 30 décembre 2012

Pagination : 61-84

ISBN : 978-2-343-00589-8

ISSN : 1157-996X

Référence électronique

Michèle Morel et Nicole de Moor, « Migrations climatiques : quel rôle pour le droit international ? », Cultures \& Conflits [En ligne], 88 | hiver 2012, mis en ligne le 15 mars 2014, consulté le 30 mars 2021. URL : http://journals.openedition.org/conflits/18580 ; DOI : https://doi.org/10.4000/conflits. 18580 


\title{
Migrations climatiques : quel rôle pour le droit international ? 1
}

\section{Michèle MOREL, Nicole de MOOR}

\begin{abstract}
Michèle Morel a fait ses études à l'Université de Gand et de Nottingham et a été chercheur invitée à l'Université d'Oxford en 2011. Elle a obtenu sa thèse de doctorat en droit à l'Université de Gand en tant que chercheur de la Fondation pour la recherche de Flandre ( $F W O$ ). Sa recherche porte sur la protection contre le déplacement arbitraire dans le droit international. Elle examine dans quelle mesure le droit de ne pas être déplacé existe en droit international.
\end{abstract}

Nicole de Moor a travaillé comme conseiller juridique en droit de l'immigration pour des agence gouvernementales et non gouvernementales. Elle mène actuellement une recherche doctorale sur les migrations environnementales à la faculté de droit de l'Université de Gand. Plus particulièrement, sa recherche porte sur les différents cadres juridiques qui pourraient faciliter la migration internationale comme stratégie d'adaptation des communautés affectées par la dégradation de l'environnement.

T e sort de ceux qui sont appelés les « réfugiés climatiques » est d'actualité ¿dans les débats et les écrits scientifiques. Géographes, anthropologues, politologues et juristes se réunissent autour de la question pour débattre de leur existence, leur nombre, les causes de leur fuite, et les cadres institutionnels politiques et juridiques existants ou nécessaires. Alors que les individus ont toujours utilisé la migration comme une stratégie pour répondre aux changements environnementaux, c'est l'attention mondiale croissante portée au phénomène du changement climatique qui a amené les universitaires, les décideurs et la communauté des ONG à s'intéresser à ceux qui sont contraints de quitter leur lieu de vie habituel en raison de changements environnementaux. Il est important de constater que beaucoup de ceux qui subissent aujourd'hui les effets de ces changements n'ont pas les moyens nécessaires leur permettant de se déplacer. En conséquence, ils risquent de se trouver

1. Texte traduit de l'anglais par Angélique Petrits. Toutes les traductions des citations anglaises sont du même traducteur. 
négligés et dans l'ombre de ceux qui sont en mouvement, malgré le fait qu'ils méritent autant d'attention et de protection. Enfin, les recherches de terrain ont également montré que les personnes migrent non seulement hors des zones à hauts risques environnementaux, mais se déplacent aussi vers des zones vulnérables telles que les zones urbaines de basse altitude, les méga-deltas ou les taudis dans des villes sujettes aux inondations ${ }^{2}$.

L'enjeu des migrations climatiques soulève des questions spécifiques et importantes, parmi lesquelles des problématiques juridiques analysées dans cet article. La littérature existante sur le sujet traite de ces questions parfois dans le cadre plus large du droit international général et parfois dans le cadre spécifique d'une branche du droit international telle que le droit de l'environnement, les droits de l'homme, des réfugiés ou le droit des migrations. Le cadre spécifique sélectionné dépend de l'approche et de la spécialisation de chaque auteur. Juristes des droits humains, juristes des réfugiés, juristes des questions migratoires ou de l'environnement adoptent chacun une approche différente et leur échanges paraissent difficiles, particulièrement en ce qui concerne les juristes des réfugiés et des migrations et les juristes de l'environnement. Comme le signale Gemenne, la recherche sur les migrations environnementales est « partagée entre ceux qui adoptent une vision alarmiste et ceux que l'on pourrait qualifier de sceptiques et reflète l'écart disciplinaire qui sépare les sciences naturelles et les sciences sociales » ${ }^{3}$. Alors que les chercheurs de l'environnement ont tendance à avoir une vision plutôt alarmiste, avertissant que le changement climatique conduira à des déplacements massifs, les spécialistes des migrations sont plus sceptiques, affirmant que le changement environnemental n'est qu'un facteur parmi d'autres du phénomène migratoire. Ces points de vue opposés ont conduits à des désaccords sur le cadre juridique nécessaire pour traiter la question des migrations climatiques et conduisent à s'interroger sur la contribution de chacune de ces branches du droit international à la protection des migrants climatiques ainsi que sur leurs convergences et leur éventuelle complémentarité.

Cet article examine le rôle de ces quatre branches du droit international le droit de l'environnement, le droit international des droits de l'homme, le droit international des réfugiés et le droit international des migrations - abordant différents aspects des migrations climatiques. Les aspects étudiés portent sur la question de la prévention de la migration forcée, la question de l'admission des migrants sur le territoire étranger, et la question des droits exerçables

2. Foresight Final Project Report on "Migration and Global Environmental Change: Future Challenges and Opportunities”, The Government Office for Science, Londres, 2011, disponible sur : http://bis.gov.uk/assets/bispartners/foresight/docs/migration/11-1116-migrationand-global-environmental-change.pdf.

3. Gemenne F., "How they became the human face of climate change. Research and policy interactions in the birth of the 'environmental migration' concept”, in Piguet, E., Pécoud A. et De Guchteneire P. (eds.), Migration and Climate Change, Unesco Publishing, Cambridge University Press, Cambridge, 2011, p. 225-259. 
dans le pays ou le lieu d'arrivée. En adoptant cette approche, il devient clair que les quatre branches du droit international ont leurs propres fonctions et contributions spécifiques dans le cas des migrations climatiques et que, par conséquent, elles sont parfaitement complémentaires.

En ce qui concerne la terminologie, cet article utilise les termes « migrations/migrants climatiques internes » et « migration/migrants climatiques externes ». Le terme « migrant » n'ayant pas de définition universelle, et par conséquent pas de signification fixe en droit international, il comprend deux mouvements : d'une part ceux réalisés à l'intérieur d'un pays et les mouvements transfrontaliers, d'autre part les mouvements forcés et volontaires. Le terme « réfugié » n'est pas utilisé en raison de son sens juridique strict ou parce que les migrants climatiques ne sont pas nécessairement des réfugiés. Dans la catégorie des déplacements forcés, des différences existent concernant la mesure de la contrainte. Certaines personnes fuient pour survivre (par exemple, en cas de catastrophe naturelle soudaine), d'autres pour échapper à la pauvreté extrême ou à des problèmes de santé liés à l'environnement. À certains égards, il peut être justifié de ne considérer que les personnes qui fuient pour survivre. Dans le cadre de l'octroi du droit d'asile sur un territoire étranger, par exemple, il paraît logique - en tenant compte de la grande pénurie des endroits d'entrée dans le monde d'aujourd'hui - de donner la priorité à ces demandeurs d'asile ayant la requête la plus légitime ou la plus convaincante en vue d'une protection internationale ${ }^{4}$.

Dans ce contexte, il convient de se référer à la notion de «migrants de survie », développée en 2009 à l'Université d'Oxford 5. Les migrants de survie sont caractérisés, entre autres, par la menace existentielle à laquelle ils sont confrontés. Fait intéressant, les raisons de leur fuite ne sont pas limitées aux changements environnementaux, mais sont principalement dues à une combinaison de facteurs environnementaux, sociaux et économiques. Dans ce cas, les auteurs insistent sur le caractère généralement multi-causal des déplacements forcés. Le but de cet article n'est pas d'identifier des mécanismes internationaux de protection pour les plus démunis mais plutôt d'analyser la pertinence des différentes branches du droit international à l'égard du problème des migrations climatiques. Il n'est ainsi pas nécessaire de classer les différents types de migrants climatiques en fonction de la gravité de la menace à laquelle ils sont confrontés. Par conséquent, le terme « migration de survie » ne sera pas utilisé. Néanmoins, lorsque nous utilisons le terme « migration clima-

4. Gibney M., The ethics and politics of asylum. Liberal democracy and the response to refugees, Cambridge, Cambridge University Press, 2004, p. 12.

5. Betts A., Kaytaz E., "National and international responses to the Zimbabwean exodus: implications for the refugee protection regime", New Issues in Refugee Research, Research Paper $\mathrm{n}^{\circ}$ 175, 2009, Genève, UNHCR. Voir aussi Morel M., Maes F., "The curious phenomenon of 'environmental migration/displacement' \& the role of international law in cross-border protection”, in French D. (ed.), Global Justice and Sustainable Development, Leiden, Brill, 2010, pp. 273-288. 
tique », les relations causales complexes, mentionnées ci-dessus, qui sont à la base de la décision de migrer, doivent être reconnues, en gardant à l'esprit que les facteurs environnementaux qui poussent à la migration (push factors) sont souvent accompagnés par d'autres éléments (par exemple, sociaux et économiques).

\section{Droit international de l'environnement}

La question peut être posée de savoir si, et dans quelle mesure, le droit international de l'environnement comporte des principes, des règles ou des dispositions qui abordent explicitement la migration climatique. Dans ce contexte, la Convention des Nations unies sur les changements climatiques (CCNUCC) ${ }^{6}$, les Accords de Cancún ${ }^{7}$ et la Convention des Nations unies sur la lutte contre la désertification dans les pays gravement touchés par la sécheresse et/ou la désertification, en particulier en Afrique (UNCCD), ${ }^{8}$ sont les textes les plus pertinents.

\section{L'émergence de la question migratoire dans le régime climatique internatio- nal}

Au moment de l'adoption de la CCNUCC en 1992, l'accent était mis sur l'atténuation et les effets néfastes du réchauffement climatique. Ni la Convention, ni son Protocole de Kyoto ${ }^{9}$ adopté en 1997 pour renforcer les obligations de limitation des émissions mondiales de gaz à effet de serre, n'ont abordé la mobilité humaine liée au climat. Comme la période d'engagement du Protocole prendra fin cette année, les parties à la Convention ont longtemps négocié les contours du régime international climatique après 2012. Alors que les négociations sur le climat étaient à l'origine exclusivement axées sur la réduction des émissions de gaz à effet de serre, les acteurs mobilisés en la matière sont devenus ensuite de plus en plus préoccupés par la dimension humaine du changement climatique et la nécessité de s'adapter pour compenser les conséquences inévitables du réchauffement climatique.

Avec la publication du quatrième rapport d'évaluation du Groupe d'experts intergouvernemental sur l'évolution du climat (GIEC) en $2007^{10}$, les

6. 9 Mai 1992, 1771 UNTS 107, 31 ILM 849.

7. UNFCCC, The Cancin Agreements: Outcome of the work of the Ad Hoc Working Group on Long-term Cooperative Action under the Convention (AWG-LCA), Decision 1/CP.16, adoptée à la Conférence des parties sur sa seizième session, tenue à Cancún du 29 novembre au 10 décembre 2010, FCCC/CP/2010/7/Add.1. Pour le texte de l'accord voir http://unfccc.int/resource/docs/2010/cop16/eng/07a01.pdf\#page=2.

8. 17 juin 1994, 1954 UNTS 3, 33 ILM 1328.

9. Protocole de Kyoto du 11 décembre 1997 à la Convention cadre des Nations Unies sur les changements climatiques, entrée en vigueur le 16 février 2005, UNTS 2303, p. 148.

10. Groupe d'Experts Intergouvernemental sur l'Évolution du Climat (GIEC), "Climate Change 2007: Synthesis Report. Summary for Policymakers”, in Fourth Assessment Report, 2007. 
discussions, dans les négociations de la CCNUCC, se sont déplacées vers des mesures d'adaptation. Comme le GIEC avait déjà mis en évidence, en 1990 11, les liens entre migration et réchauffement climatique, certaines organisations internationales, gouvernementales et non gouvernementales, accompagnées d'universitaires et certains pays en développement, ont exercé des pressions pendant deux décennies pour introduire la question de la mobilité humaine liée au climat dans le cadre de la CCNUCC ${ }^{12}$. Selon l'Organisation internationale pour les migrations (OIM), la migration doit être pleinement intégrée dans le cadre de l'adaptation au changement climatique ${ }^{13}$.

S’appuyant sur une base de connaissances émergentes sur la migration environnementale, la communauté humanitaire a finalement réussi à mettre la mobilité climatique sur la table des négociations. À partir de 2008, les migrations climatiques sont apparues dans les différentes versions du texte de négociation. Lors de la 16ème session de la Conférence des Parties (COP 16) à Cancùn en décembre 2010, les parties ont réussi à conclure un accord modeste faisant apparaître pour la première fois la question des migrations climatiques dans une décision officielle du COP ${ }^{14}$. Un cadre visant à renforcer les mesures d'adaptation 15 est adopté. Il comprend l'alinéa 14 (f) sur la migration climatique, qui énonce :

«14. Invite toutes les Parties à renforcer l'action sur l'adaptation au titre du Cadre d'adaptation de Cancùn, en tenant compte de leurs responsabilités communes mais différenciées, de leurs capacités res-

11. Groupe d'experts intergouvernemental sur l'évolution du climat (GIEC), First Assessment Report, Groupe de travail II : Impacts Assessment of Climate Change, 1990.

12. Kolmannskog V., "Climate Changed, People Displaced”, Thematic Report from the Norwegian Refugee Council, 2009 ; Lawton C., "'What about Climate Refugees? Efforts to Help the Displaced Bog Down in Copenhagen”, Spiegel Online, 17 December 2009, disponible sur : http://www.spiegel.de/international/europe/0,1518,druck-667256,00.html ; voir aussi Warner K., "Climate Change Induced Displacement: Adaptation Policy in the Context of the UNFCCC Climate Negotiations”, document préparé pour la Table ronde d'experts du HCR sur les changements climatiques et les déplacements, 22-25 Février 2011 à Bellagio, Italie, Legal and Protection Policy Research Series, UNHCR, Division of International Protection, mai 2011, disponible sur : http://www.unhcr.org/refworld/docid/4e09a3d32.html ; Warner K., "PD23: Migration and displacement in the context of adaptation to climate change: developments in the UNFCCC climate negotiations and potential for future action", Étude commandée dans le cadre du projet Foresight du Gouvernement britanique, Migration and Global Environmental Change, octobre 2011, disponible sur : http://bis.gov.uk/assets/bispartners/foresight/docs/migration/policy-development/11-1269-pd23-migration-displacement-in-adaptation-climatechange.pdf (cité as Warner K. (2011b)).

13. Organisation Internationale pour les Migrations (OIM), "Disaster risk reduction, climate change adaptation and environmental migration: A Policy Perspective”, Genève, 2010, disponible sur : http://publications.iom.int/bookstore/free/DDR_CCA_report.pdf.

14. UNFCCC, The Cancin Agreements: Outcome of the work of the Ad Hoc Working Group on Long-term Cooperative Action under the Convention (AWG-LCA), Decision 1/CP.16, adoptée à la Conférence des Parties sur sa seizième session, tenue à Cancún du 29 novembre au 10 décembre 2010, FCCC/CP/2010/7/Add.1.

15. Rajamani L., "The Cancún Climate Agreements: Reading the Text, Subtext and Tea Leaves”, International and Comparative Law Quarterly, 60, 2011, pp. 499-519. 
pectives, et de leurs priorités spécifiques nationales et régionales en matière de développement, de leurs objectifs et selon les circonstances, en s'engageant, inter alia, sur les points suivants :

(f) Mesures visant à améliorer la compréhension, la coordination et la coopération en matière de déplacement, migration et réinstallation planifiée induits par le changement climatique, le cas échéant, aux niveaux national, régional et international ${ }^{16}$, [nous soulignons]

Alors qu'en 2009, l'ébauche d'un texte de négociation se référait toujours aux « réfugiés climatiques », l'alinéa 14 (f) introduit une approche plus nuancée en présentant divers types de mobilité humaine qui exige toute une gamme de mesures en vue d'une mise en ouvre à différents niveaux ${ }^{17}$. Il invite les États à adopter une approche proactive et à aborder les questions migratoires traditionnelles dans les politiques publiques ${ }^{18}$. L'avenir devra préciser quel type de mesures pourrait émerger de ce paragraphe. Les États sont invités à soutenir davantage la recherche sur la migration climatique, tant au niveau national que régional ou international. Selon Warner, la coordination en matière de déplacements continuera à avoir lieu au sein des organisations humanitaires, tandis que la coordination en matière de réinstallation planifiée, étant encore à ses balbutiements, pourrait commencer à être discutée par les pays qui adoptent des projets d'adaptation nécessitant la réinstallation des populations. Dans l'avenir, une coopération plus opérationnelle sera nécessaire, en particulier lorsque le changement climatique génère des flux plus importants de personnes déplacées. Des exemples de migrations transfrontalières effectuées suite à des accords temporaires en matière de migration de main-d'œuvre pourraient servir de modèles pour une coopération opérationnelle en matière de migration comme stratégie d'adaptation ${ }^{19}$.

L'inclusion de la mobilité humaine dans le Cadre de Cancún pour l'adaptation représente une étape importante pour la question des migrations environnementales. C'est la première fois que la question migratoire est mentionnée dans un document décidé par le COP. Bien que les Accords de Cancún ne soient pas juridiquement contraignants, l'alinéa 14 (f) a une forte valeur politique, car il est situé dans une partie importante du Cadre pour l'adaptation. Les paragraphes 13 et 14 fournissent une liste d'activités qui peuvent être considérées comme relevant de "l'adaptation », et pourraient donc bénéficier des fonds d'adaptation dans l'avenir 20. Le fait qu'un financement pour les migrations puisse être disponible au sein de la CCNUCC est l'une des spécificités les plus importantes de ce Cadre par rapport à d'autres cadres juridiques internationaux portant sur la mobilité humaine.

16. Paragraphe 14(f) de l'accord de Cancùn, voir note 14.

17. Warner K., "PD23: Migration and displacement in the context of adaptation to climate change...", op. cit.

18. Ibid.

19. Warner K, op. cit.

20. Ibid. 
Outre l'amélioration des possibilités de financement, aborder les migrations dans le cadre international sur le climat a l'avantage de donner « une voix à toutes les nations du monde ", y compris les pays les plus vulnérables, dans un des plus importants forums internationaux ${ }^{21}$. Le paragraphe 14 (f) du Cadre de Cancún pourrait même encourager les instances politiques d'autres forums à aborder la question des migrations environnementales aux niveaux national, régional et international 22. Toutefois, comme indiqué auparavant, les engagements convenus dans le Cadre de Cancún sont volontaires et non contraignants. Bien que le paragraphe 14 (f) invite les États à prendre des mesures concernant la compréhension, la coordination et la coopération relatives à la mobilité humaine induite par le changement climatique, il ne les invite pas à accorder des droits de résidence aux personnes déplacées en raison du climat, ou à faciliter la migration internationale pour les populations les plus touchées. En effet, la migration internationale touche à l'importante prérogative nationale de définir qui peut entrer sur le territoire d'un pays. Que les États décident ou non de faciliter la migration internationale en tant que mesure d'adaptation reste donc une affaire de souveraineté nationale qui doit être traitée par la loi et la politique migratoire aux niveaux national ou régional et non pas par le droit international de l'environnement. Enfin, le régime de la CCNUCC ne peut aborder que les migrations dues au climat, sans tenir compte d'autres facteurs environnementaux les favorisant au départ.

Les Accords de Cancún ne marquent pas la fin des négociations sur le régime climatique après 2012. Certaines des prérogatives convenues à Cancùn ne peuvent être pleinement réalisées qu'après avoir précisé davantage de nombreux détails de cette initiative ${ }^{23}$. Bien que la 17 e session de la Conférence des Parties (COP 17) à Durban, en novembre/décembre 2011 ait soutenu la mise en œuvre du Cadre de Cancún avec un certain nombre de décisions portant sur l'adaptation, une série de dispositions doit encore être développée pour permettre la mise en œuvre du paragraphe 14 (f).

Les migrations environnementales dans la Convention des Nations Unies sur la lutte contre la désertification (UNCCD)

Bien que ni la CCNUCC ni le Protocole de Kyoto ne se réfèrent explicitement au phénomène du changement climatique ou à celui des migrations environnementales, la UNCCD leur accorde une attention modeste:

Ayant à l'esprit que la désertification et la sécheresse compromettent le développement durable en raison de la corrélation qui existe entre ces phénomènes et d'importants problèmes sociaux, comme la pauvreté, une mauvaise situation sanitaire et nutritionnelle et l'insé-

21. Ibidem.

22. Ibidem.

23. Rajamani L., op. cit. 
curité alimentaire, ainsi que ceux qui découlent des migrations, des déplacement de personnes et de la dynamique démographique. (Préambule)

Les programmes d'action nationaux peuvent inclure [...] tout ou partie des mesures suivantes pour se préparer à atténuer les effets de la sécheresse : (a) la création et/ou le renforcement, le cas échéant, de systèmes d'alerte précoce [...] ainsi que des mécanismes pour aider les personnes déplacées pour des raisons écologiques ${ }^{24}$. (Article 10), [nous soulignons]

Les Parties s'engagent, selon leurs capacités respectives, à favoriser la coopération technique et scientifique dans les domaines de la lutte contre la désertification et de l'atténuation des effets de la sécheresse. [...] À cet effet, elles appuient les activités de recherche qui : [...] tiennent compte, lorsqu'il y a lieu, des rapports entre la pauvreté, les migrations dues à des facteurs écologiques 25 et la désertification. (Article 17) [nous soulignons]

\section{Le rôle préventif du droit international de l'environnement}

Plutôt que d'adopter une approche explicite, le droit de l'environnement joue implicitement un rôle important dans le traitement des migrations climatiques. Le droit international de l'environnement est une branche du droit international qui vise à protéger l'environnement naturel pouvant être affecté par l'activité humaine ${ }^{26}$. Compte tenu de la dépendance des êtres humains à l'égard de l'environnement, surtout dans certaines régions et pour certaines populations en raison de leur mode de vie spécifique, la protection de l'environnement naturel contribue souvent à la protection des moyens de subsistance des êtres humains 27 . Le droit international de l'environnement joue ainsi un rôle dans la protection des moyens de subsistance et, plus généralement, dans la protection des droits de l'homme. Par conséquent, cette branche $\mathrm{du}$ droit international est tout à fait pertinente dans le cadre de la prévention des migrations climatiques. À titre d'exemple, dans le contexte du changement climatique, nous pouvons évoquer les initiatives et actions en matière d'atténuation et d'adaptation abordées dans la CCNUCC, le protocole de Kyoto et les Accords de Cancún. Une des causes des migrations climatiques est abordée à travers les mesures d'atténuation de changement climatique. L'adaptation au changement climatique, dans la mesure où elle vise à réduire les impacts actuels du changement climatique et cherche à accroître la rési-

24. Nous ajoutons l'italique.

25. Nous ajoutons l'italique.

26. Sands P., Principles of international environmental law, Cambridge, Cambridge University Press, 2003, p. 3.

27. La protection de l'environnement peut également avoir un impact négatif sur les moyens de subsistance des personnes. Par exemple, on peut citer la situation où la conservation de l'environnement, sous la forme de la désignation de zones de réserves naturelles, provoque l'expulsion des tribus indigènes de leur terre (voir l'article de J. Shade dans ce numéro). 
lience des populations aux impacts futurs, contribue à la protection de la vie de la population et donc à la prévention des migrations climatiques ${ }^{28}$.

\section{Le droit international des droits de l'Homme}

Le droit international des droits de l'Homme est potentiellement pertinent pour traiter le problème des migrations climatiques et ce de trois manières. Tout d'abord, il précise les droits humains des potentiels migrants climatiques dans leur pays d'origine ou de résidence habituelle. Les instruments les plus intéressants relatifs aux droits de l'homme à cet égard sont les suivants : la Déclaration universelle des droits de l'Homme de 1948 (DUDH) ${ }^{29}$; la première expression universelle des droits qui concerne tous les êtres humains, le Pacte international de 1966 relatif aux droits civils et politiques (PIDCP) 30, stipulant aux articles 6, 7 et 1 (2) respectivement le droit à la vie, le droit de ne pas être soumis à des traitements cruels, inhumains ou dégradants et le droit de ne pas être privé de moyens de subsistance; et le Pacte international de 1966 relatif aux droits économiques, sociaux et culturels (PIDESC) ${ }^{31}$, qui dans l'article 11 protège, entre autres, le droit à un niveau de vie suffisant, y compris les besoins en nourriture et en logement. D'autres instruments qui offrent une protection des droits humains à des groupes sociaux spécifiques, sont la Convention de 1989 sur les droits de l'enfant (CRC) ${ }^{32}$, la Convention de 1979 sur l'élimination de toutes les formes de discrimination à l'égard des femmes (CEDAW) ${ }^{33}$ et, en 1989, la Convention de l'OIT sur les droits des peuples autochtones ${ }^{34}$.

Si les droits de l'Homme avaient été effectivement appliqués, il y aurait moins de migrations forcées dans la mesure où le déplacement implique, dans la plupart des cas, une violation des droits humains. La législation sur les droits de l'Homme joue donc un rôle important dans la prévention de la migration climatique. Cependant, tous les États ne sont pas parties de l'ensemble des instruments internationaux relatifs aux droits de l'Homme et, pour ceux qui ont accepté d'être liés par les obligations internationales, le manque de volonté ou l'impuissance des gouvernements nationaux ont souvent eu pour conséquence une mise en œuvre insuffisante. Par conséquent, l'efficacité pratique du droit international des droits de l'Homme, en tant que source de

28. Le Fonds d'adaptation, financé par une part du produit du Mécanisme de développement propre et supervisé par le Conseil du fonds pour l'adaptation, finance des projets concrets dans les pays en développement qui sont parties au Protocole de Kyoto et particulièrement vulnérables aux effets négatifs du changement climatique. Voir : http://www.adaptationfund.org/.

29. 10 décembre 1948, GA Res. 217 (III), UN GAOR, 3d Sess., Supp. No. 13, UN Doc. A/810 (1948) 71.

30. 19 décembre 1966, 999 UNTS 171.

31. 19 décembre 1966, 993 UNTS 3.

32. 20 novembre 1989,1577 UNTS 3.

33. 18 décembre 1979, 1249 UNTS 13.

34. 27 juin 1989, 1650 UNTS 384. 
droits que les individus peuvent, en théorie, exercer dans leur pays d'origine ou de résidence habituelle pour lutter contre le problème de la migration climatique, ne doit pas être surestimée. Le renforcement des droits de l'Homme par les gouvernements nationaux est nécessaire pour renforcer la prévention de la migration forcée.

Les droits de l'Homme peuvent également être utiles pour les migrants du climat dans le cadre des migrations climatiques externes. Le droit international des droits humains, s'appliquant à chaque personne sans discrimination, est applicable aux étrangers (le cas échéant, aux migrants climatiques qui ont traversé une frontière internationale) de la même manière qu'il l'est pour les citoyens. Les deux types de migrants, légaux ou réguliers et clandestins ou irréguliers sans titre de séjour, - par exemple, demandeurs d'asile déboutés ou ceux qui parviennent à entrer et à rester dans un pays étranger sans-papiers et sans faire une demande d'asile - jouissent des droits fondamentaux consacrés en droit international des droits de l'Homme. Une catégorie spécifique de personnes, situées entre les migrants réguliers et les migrants irréguliers, ne relèvent pas du cadre international des réfugiés mais sont néanmoins «non refoulables » : ce sont les personnes qui ne peuvent pas être légalement renvoyées dans leur pays d'origine ou de résidence habituelle (c'est le principe de nonrefoulement, qui est discuté ci-après). Le cas d'une menace de torture constitue un bon exemple. Cette catégorie d'individus relève également du droit international des droits de l'Homme.

Les migrants climatiques ayant franchi une frontière internationale et se trouvant sur un territoire étranger profitent ainsi de tous les droits fondamentaux, indépendamment de leur situation (régulière ou non). Cependant, dans la pratique, les migrants irréguliers sont souvent privés de soins médicaux, de logement et d'autres services sociaux. La détention de demandeurs d'asile déboutés est une pratique courante et ils sont souvent traités comme des criminels ${ }^{35}$. Dans le climat mondial actuel, les migrants sont considérés comme une menace pour la culture, l'économie nationale, et la sécurité. La mise en œuvre du droit international des droits humains, en tant que source de droits, qui reconnaît que les individus peuvent jouir de tous ces services non seulement dans leur pays d'origine ou de résidence habituelle, mais aussi dans un pays étranger où ils ont dû fuir, doit être renforcée pour permettre à cette branche du droit de contribuer plus efficacement à la protection des migrants climatiques externes. Il convient de mentionner dans ce contexte la Déclaration de 1985 sur les droits des personnes qui ne possèdent pas la nationalité du pays dans lequel elles vivent ${ }^{36}$, adoptée par l'Assemblée générale des Nations unies. Cette déclaration énumère un certain nombre de droits dont les non-ressortissants jouissent dans l'État où ils se trouvent. Alors qu'un pre-

35. Weissbrodt D., The human rights of non-citizens, Oxford, Oxford University Press, 2008, pp. 147-148.

36. 13 décembre 1985, A/RES/40/144. 
mier ensemble de droits (y compris le droit à la vie, à l'interdiction de la torture, à la vie privée et au domicile, et à la liberté de pensée) est destiné à tous ceux qui ne disposent pas de la nationalité, qu'ils soient réguliers ou irréguliers, certains droits supplémentaires sont exclusivement réservés aux étrangers résidant légalement dans un pays (par exemple, le droit aux soins médicaux, le droit à une vie sûre, à la santé, au travail, etc.).

Enfin, le droit international des droits de l'Homme peut être pertinent dans le contexte de l'admission de personnes dans un pays étranger. Le droit d'être protégé contre un retour forcé, connu sous le nom du principe de nonrefoulement, interdit à un État de renvoyer, expulser ou extrader une personne de quelque manière que ce soit vers un autre État où cette personne risque d'être soumise à de graves violations des droits de l'Homme ${ }^{37}$. Il est nécessaire de savoir si ce principe pourrait protéger les personnes déplacées qui ont franchi une frontière internationale en raison des changements climatiques. Cette question ayant été analysée en détail 38 , il convient de souligner que, sur la base de l'interprétation des dispositions des conventions internationales et régionales ${ }^{39}$ ainsi que sur celle de la jurisprudence de la Cour européenne des droits de l'homme ${ }^{40}$, les migrants climatiques qui ont franchi une frontière internationale ne sont actuellement pas protégés par le principe de non-refoulement. En effet, ce principe ne traite pas tous les types de personnes déplacées de manière équitable. C'est pourquoi, tout État peut légalement les renvoyer dans leur pays d'origine ou de résidence habituelle, à condition qu'ils ne soient pas protégés par un autre cadre juridique.

Par conséquent, à l'heure actuelle, le droit international des droits de l'Homme n'est pas pertinent dans ce contexte. Toutefois, il n'est pas totalement exclu que la jurisprudence à venir, élaborée par des organismes internationaux ou régionaux, définisse le retour d'une personne dans une région où elle risque de faire face à des problèmes environnementaux extrêmement sévères (avec un pronostic vital engagé), comme une violation des droits de l'homme (en se basant, par exemple, sur le droit à la vie ou à la liberté face à un traitement inhumain).

37. Wouters K., International legal standards for the protection from refoulement, Mortsel, Intersentia, 2009.

38. Morel M. et Maes F., "The curious phenomenon of 'environmental migration/displacement" and the role of international law in cross-border protection“, in French D. (ed.), Global Justice and Sustainable Development, Leiden, Brill, 2010, pp. 273-288.

39. La Convention relative au statut des réfugiés, 28 juillet 1951, 189 UNTS 137, modifiée par le Protocole relatif au statut des réfugiés, 31 janvier 1967, 606 UNTS 267 (1951, Convention sur les réfugiés), la Convention des Nations Unies contre la torture et autres peines ou traitements cruels, inhumains ou dégradants (CCT), 10 Décembre 1984, 1465 UNTS 85, la Convention européenne pour la protection des droits de l'homme et des libertés fondamentales (CEDH), 213 UNTS 221, et le PIDCP, voir note 29.

40. D v. the United Kingdom (1997) 24 EHRR 423; N v. the United Kingdom (2008) 47 EHRR 885 . 


\section{Droit international des réfugiés}

Le droit international des réfugiés, au sens large du terme ${ }^{41}$, est un ensemble de règles et de procédures visant à protéger les personnes qui cherchent l'asile dans un pays étranger après avoir été forcées de quitter leur pays d'origine pour certaines raisons précisées dans des instruments juridiques ad hoc. La protection légale accordée par la loi internationale sur les réfugiés aux différents groupes de personnes recouvre partiellement le droit international des droits de l'Homme. Néanmoins, alors que le régime des droits humains a un rôle normatif ${ }^{42}$, le droit international des réfugiés traite des situations dans lesquelles « la seule véritable solution pour un être humain » est « le départ lorsque les circonstances deviennent intolérables 43 », ces circonstances équivalant à une violation des droits de l'Homme. Le droit des réfugiés a donc un rôle palliatif ${ }^{44}$.

Le cadre juridique international pour les migrants forcés qui ont traversé une frontière internationale se compose d'un instrument international, la Convention relative au statut des réfugiés (Convention de 1951) ${ }^{45}$, et quatre instruments régionaux : la Convention régissant les aspects propres aux problèmes des réfugiés en Afrique (Convention de l'OUA, 1969) 46, la Déclaration de Carthagène sur les réfugiés de l'Organisation des États américains (Déclaration de l'OEA, 1984) 47, la directive 2004/83/CE du 29 avril $2004 \mathrm{du}$ Conseil de l'UE sur les normes minimales pour la qualification et le statut de ressortissants de pays tiers ou des apatrides ou des personnes qui, autrement, ont un besoin de protection internationale et sur le contenu de la protection à accorder (Directive de qualification de l'UE) ${ }^{48}$, et enfin la directive 2001/55/CE du 20 juillet 2001 du Conseil de l'UE sur les normes minimales pour l'octroi d'une protection temporaire en cas d'afflux massif de personnes déplacées et sur les mesures visant à promouvoir un effort équilibré entre les États membres sur l'accueil de ces personnes et sur le poids des conséquences de ceci (Directive européenne sur la protection temporaire) ${ }^{49}$.

41. Dans le sens étroit du terme, un « réfugié » est une personne répondant aux critères mentionnés à l'article 1 (a)(2) de la Convention de 1951 sur les réfugiés.

42. Article 2(1) PIDCP : les États parties (...) s'engagent à respecter et à garantir à tous les individus (...) les droits reconnus dans le présent Pacte; article 2(1) PIDESC : chacun des États parties (...) s'engage à agir (...) au maximum de ses ressources disponibles, en vue d'assurer progressivement le plein exercice des droits reconnus dans le Pacte.

43. Hathaway J., "New directions to avoid hard problems: The distortion of the palliative role of refugee protection”, Journal of Refugee Studies, 8, 3, 1995, pp. 293-294.

44. Association internationale des Juges de droit des réfugiés, "The intersection of human rights law and refugee law: on or off the map? The challenge of locating Appellant S395/2002Australia/", New Zealand Chapter Meeting, Sydney, 9 juin 2004, www.refugee.org.nz/Reference/Sydney04.html.

45. Voir note 38.

46. Organisation de l'Unité africaine, 10 septembre 1969, 1001 UNTS 45.

47. Colloque sur la protection internationale des réfugiés en Amérique centrale, Organization of American States, Mexico and Panama, 22 November 1984.

48. Journal Officiel L $304,30 / 09 / 2004$ p. 12-23.

49. Journal Officiel L 212, 07/08/2001 p. 12-23. 
Ces instruments relatifs aux réfugiés portent à la fois sur les critères d'admission dans un pays étranger et, bien que de manière inégale, sur les droits qui peuvent être accordés dans le pays d'accueil. Le principe du non-refoulement discuté ci-dessus, en revanche, ne traite que de l'interdiction d'un renvoi, mais pas d'autres types de droits.

La Convention de 1951 définit un « réfugié » pouvant prétendre à une protection juridique comme une personne qui :

craignant avec raison d'être persécutée du fait de sa race, de sa religion, de sa nationalité, de son appartenance à un groupe social ou de ses opinions politiques, se trouve hors du pays dont elle a la nationalité et qui ne peut ou, du fait de cette crainte, ne veut se réclamer de la protection de ce pays ; ou qui, si elle n'a pas de nationalité et se trouve hors du pays dans lequel elle avait sa résidence habituelle à la suite de tels événements, ne peut ou, en raison de ladite crainte, ne veut y retourner (Article 1 (a) (2))

De nombreuses recherches ont été réalisées sur l'applicabilité de la Convention de 1951 dans le cas de migrations climatiques ${ }^{50}$. Il est désormais largement admis que la Convention de 1951 n'est pas applicable : pour satisfaire les critères de cette Convention, la persécution doit émaner d'un auteur et doit être liée à l'un des cinq motifs de la Convention. Certains ont fait valoir que les migrants climatiques constituent un « groupe social particulier »-l'un des cinq motifs de la Convention - composé de personnes n'ayant pas le pouvoir politique nécessaire pour protéger leur environnement ${ }^{51}$. Toutefois, cette interprétation est controversée et ne semble pas constituer la bonne approche. L’impuissance politique ne peut pas être considérée comme une caractéristique permanente qui permet de classer quelqu'un comme membre d'un

50. Par exemple, Cooper J., "Environmental refugees: meeting the requirements of the refugee definition”, New York University Environmental Law Journal, 6, 1998, pp. 480-529; Falstrom D., "Stemming the flow of environmental displacement: creating a convention to protect persons and preserve the environment", Colorado Journal of International Environmental Law and Policy, 1, 6, 2001, pp. 1-32 ; Aleinikoff A., "Protected characteristics and social perceptions: an analysis of the meaning of "membership of a particular social group" "' in Feller E., Türk V. et Nicholson F. (eds.), Refugee Protection in International Law, Cambridge, Cambridge University Press, 2003, pp. 263-311 ; McAdam J., "Climate change "refugees" and international law", NSW Bar Association, 24 octobre 2007, www.nswbar.asn.au/circulars/climatechange1.pdf ; McAdam J., "Climate Change Displacement and International Law: Complementary Protection Standards”, Document préparé pour la Table ronde d'experts du HCR sur les changements climatiques et les déplacements, 22-25 février 2011 à Bellagio, en Italie, Legal and Protection Policy Research Series, UNHCR, Division of International Protection, May 2011, disponible sur : http://www.unhcr.org/refworld/docid/4e09a3492.html; Williams A., "Turning the tide: recognizing climate change refugees in international law", Law E Policy, 30, 4, 2008, pp. 502529 ; Cournil C., “The protection of 'environmental refugees' in international law” in Piguet E., Pécoud A. et De Guchteneire P., Migration and Climate Change, Unesco Publishing, Cambridge University Press, Cambridge, 2011, pp. 359-387.

51. Cooper J., op. cit. 
groupe social donné et, de la même manière, la notion de persécution ne peut être utilisée pour définir le groupe social ${ }^{52}$.

Pour autant, les migrants climatiques peuvent être considérés comme des réfugiés dans la mesure où une personne fuyant des problèmes environnementaux peut craindre aussi de subir une persécution au titre de l'un des cinq motifs de la Convention. Dans ce cas, il bénéficie de la protection de la Convention de 1951, comme par exemple lorsqu'un gouvernement refuse volontairement d'offrir assistance à un groupe minoritaire après qu'un cyclone ait touché le pays. Cependant, ce cas de figure constitue plutôt l'exception que la règle.

La Convention de 1969 de l'OUA, qui traite de la migration forcée en Afrique, prévoit dans son article 1 (2), après avoir réaffirmé la définition du réfugié de 1951 dans son article 1 (1), que le terme "réfugié » doit également s'appliquer à...

...toute personne qui, du fait d'une agression, d'une occupation extérieure, d'une domination étrangère ou d'événements troublant gravement l'ordre public 53 dans une partie ou dans la totalité de son pays d'origine ou du pays dont elle a la nationalité, est obligée de quitter sa résidence habituelle pour chercher refuge à l'extérieur de son pays d'origine ou du pays dont elle a la nationalité.

Cette définition laisse entendre que les problèmes environnementaux globaux peuvent être inclus, car ils peuvent être considérés comme des événements qui portent gravement atteinte à l'ordre public ${ }^{54}$. Toutefois, la définition du réfugié selon l'OUA pose de sérieuses difficultés d'interprétation : il y a peu ou pas de travaux préparatoires, de jurisprudence, ou de littérature académique sur lesquels s'appuyer ${ }^{55}$. Bien qu'il soit probable que les États offrent refuge aux étrangers dont les situations nécessitent une protection temporaire, ceci est beaucoup moins évident lorsqu'une protection permanente ou de long terme est nécessaire.

52. Falstrom D., op. cit.

53. Nous ajoutons l'italique.

54. Rwelamira M., "Two decades of the 1969 OAU Convention Governing the Specific Aspects of Refugee Problems in Africa”, International Journal of Refugee Law, 1, 1989, pp. 557-561 ; Lopez A., "The protection of environmentally-displaced persons in international law", Environmental Law Review, 37, 2007, pp. 365-409.

55. Shacknove A., "Who is a refugee?", Ethics, 95, 2, 1985, pp. 274-284 ; Okoth-Obbo G., "Thirty years on: a legal review of the 1969 OAU Refugee Convention governing the Specific Aspects of Refugee Problems in Africa”, Refugee Survey Quarterly, 20, 1, 2001, pp. 79-138; Rankin M., "Extending the limits or narrowing the scope? Deconstructing the OAU refugee definition thirty years on", New Issues in Refugee Research, Working Paper $n^{\circ} 113,2005$, Genève, UNHCR. 
En Amérique latine, la Déclaration de Carthagène en 1984 de l'OEA, qui traite de la migration forcée, prévoit dans son article 3 que :

le concept de réfugié utilisé dans la région englobe non seulement les éléments de la Convention de 1951 et du Protocole de 1967 mais aussi s'étend aux personnes qui fuient leur pays parce que leur vie, leur sécurité ou leur liberté étaient menacées par une violence généralisée, une agression étrangère, des conflits internes, une violation massive des droits de l'homme ou d'autres circonstances ayant perturbé gravement l'ordre public 56.

Comme dans le cas de la Convention de 1969 de l'OUA, on peut raisonnablement conclure que la migration climatique est implicitement inclue dans cette définition étant donnée la référence aux circonstances portant gravement atteinte à l'ordre public. Toutefois, dans un rapport de la Conférence internationale sur les réfugiés centraméricains (CIREFCA), qui interprète la Déclaration de Carthagène de 1984 de l'OEA, une distinction est faite entre les «catastrophes naturelles » et les autres événements qui «perturbent sérieusement l'ordre public », par exemple, des catastrophes d'origine humaine telles que celles générées par la construction de barrages, après quoi il est observé que les victimes de la première catégorie d'événements ne sont pas considérées comme des réfugiés 57 . Il convient également de noter que la Déclaration de Carthagène de 1984 de l'OEA n'est juridiquement contraignante que pour les États membres de l'Organisation des États américains. Il s'agit plutôt d'un document politique ${ }^{58}$ qui a servi de source d'inspiration pour la législation nationale sur l'asile en Amérique latine ${ }^{59}$.

En Europe, les régimes complémentaires de protection ont été développés au niveau régional afin de protéger les catégories de personnes forcées de se déplacer qui ne correspondent pas à la définition des réfugiés de 1951 mais qui, néanmoins, méritent une protection internationale. La directive sur la qualification de l'UE offre une «protection subsidiaire » dans l'Union européenne pour les personnes qui, «autrement », ont besoin d'une «protection internationale», cet « autrement » renvoyant au besoin d'une protection internationale autre que celle prévue par la Convention de 1951 (c'est à dire, la persécution pour l'un des cinq motifs évoqués). Conformément à l'article 2 (e), une personne qui est admissible à la protection subsidiaire est :

tout ressortissant d'un pays tiers ou tout apatride qui ne peut être considéré comme un réfugié, mais pour lequel il y a des motifs

56. Nous ajoutons l'italique.

57. Lopez A., op. cit.

58. Miranda C., "Toward a broader definition of refugee: 20 th century development trends", California Western International Law Journal, 20(2), 1990, pp. 315-327.

59. Voir http://www.unhcr.org/pages/49e45be46.html. 
sérieux et avérés de croire que la personne concernée, si elle était renvoyée dans son pays d'origine ou, dans le cas d'un apatride, dans le pays dans lequel il avait sa résidence habituelle, courrait un risque réel de subir les atteintes graves définies à l'article 15, l'article 17, paragraphes 1 et 2, n'étant pas applicable à cette personne, et cette personne ne pouvant pas ou, compte tenu de ce risque, n'étant pas disposée à se prévaloir de la protection de ce pays.

L'article 15 définit en outre une « atteinte grave » comme suit :

(a) la peine de mort ou l'exécution, ou (b) la torture ou des traitements ou sanctions inhumains ou dégradants infligés à un demandeur dans son pays d'origine, ou (c) des menaces graves et individuelles contre la vie ou la personne d'un civil en raison d'une violence aveugle ou en cas de conflit armé interne ou international.

Cette liste de différents types de « préjudice grave » est exhaustive et n’inclut pas explicitement le préjudice causé par les problèmes environnementaux globaux. Toutefois, la directive pourrait se révéler utile pour les migrants du climat, surtout compte tenu de la modification de la directive et de l'attention accrue portée à la relation entre changement climatique et migration (voir cidessous). En outre, on peut se poser la question si et dans quelle mesure les problèmes environnementaux graves correspondent à une torture ou des traitements inhumains comme énoncé à l'article 15 (b). Le principe précité de non-refoulement interroge sur le renvoi d'une personne vers un endroit caractérisé par des conditions environnementales difficiles et en quoi cela peut constituer une violation de l'interdiction de la torture ou d'autres traitements prohibés.

Enfin, la directive européenne sur la protection temporaire, offrant une protection temporaire dans l'Union européenne en cas d'afflux massif imminent de personnes déplacées, définit ces personnes à l'article 2 (c) en tant que :

ressortissants de pays tiers ou apatrides qui ont dû quitter leur pays ou région d'origine ou ont été évacués, notamment à la suite d'un appel lancé par des organisations internationales, dont le retour dans des conditions sûres et durables est impossible en raison de la situation régnant dans ce pays, et qui peuvent éventuellement relever du champ d'application de l'article $1 \mathrm{~A}$ de la convention de Genève ou d'autres instruments internationaux ou nationaux de protection internationale, et en particulier: i) les personnes qui ont fui des zones de conflit armé ou de violence endémique; ii) les personnes qui ont été victimes de violations systématiques ou généralisées des droits de l'homme (mise en évidence par les auteures) 
La formule « en particulier » indique que les victimes d'autres situations que les situations évoquées, par exemple en cas de catastrophe environnementale, peuvent bénéficier d'une protection temporaire, cette dernière étant définie à l'article 2 (a) de la façon suivante :

une procédure de caractère exceptionnel assurant, en cas d'afflux massif de personnes déplacées en provenance de pays tiers qui ne peuvent rentrer dans leur pays d'origine, une protection immédiate et temporaire à ces personnes, notamment si le système d'asile risque également de ne pouvoir traiter cet afflux sans provoquer d'effets contraires à son bon fonctionnement, dans l'intérêt des personnes concernées et celui des autres personnes demandant une protection.

Les personnes bénéficiant d'une protection temporaire ont le droit, pour une durée n'excédant pas celle de la protection temporaire, d'exercer une activité salariée et de suivre une formation (article 12). La durée maximale possible de la protection temporaire est de trois ans (article 4). Par conséquent, aucune protection permanente n'est prévue. Une des faiblesses de la directive est l'exigence d' «afflux massif ». Elle n'est ainsi pas applicable lorsqu'un individu a besoin de protection mais ne se trouve pas dans une situation d'afflux massif. En outre, elle ne donne aucune définition de ce terme. L'existence d'un afflux massif devrait donc être établie par une décision du Conseil (article 5). L'application de la directive sur la protection temporaire dans une situation particulière dépend donc d'une décision politique. Comme la directive n'a, jusqu'à présent, jamais été invoquée, même pas dans le cas d'une situation de conflit armé, il est hautement improbable qu'elle soit appliquée dans le cas d'une crise environnementale 60 .

Dans la mesure où la migration de l'environnement n'est pas actuellement une priorité politique pour l'Union européenne ${ }^{61}$, il ne semble pas qu'il y ait de place pour les déplacements climatiques dans le système européen actuel de l'asile. Toutefois, l'Union s'intéresse peu à peu à la question. Dans un document conjoint sur les "Changements climatiques et sécurité internationale » de 2008, la Commission européenne, en collaboration avec le Haut représentant, a évoqué la migration comme étant un résultat du changement climatique ${ }^{62}$. La Commission a recommandé de considérer les facteurs environne-

60. Kolmannskog V. et Myrstad F., "Environmental Displacement in European Asylum Law”, European Journal of Migration and Law, 11, 2009, pp. 313-326.

61. Somerville W., "PD17: Environmental migration governance: debate in the European Union”, Étude commandée dans le cadre du projet Foresight du Gouvernement britanique, Migration and Global Environmental Change, octobre 2011, disponible sur : http://bis.gov.uk/assets/bispartners/foresight/docs/migration/policy-development/11-1151pd17-environmental-migration-governance-european-union.pdf.

62. Commission Européenne (CE), Paper from the High Representative and the European Commission to the European Council on "Climate Change and International Security", 
mentaux dans le développement d'une politique européenne globale des migrations. Dans le programme de Stockholm de 2009, le Conseil européen a invité la Commission à " présenter une analyse des effets du changement climatique sur les migrations internationales, y compris ses effets potentiels sur l'immigration dans l'Union 63 ». La Commission a ensuite organisé une consultation ciblée sur les migrations liées au climat, dont le résultat est censé être inclus dans le dossier de communication sur la révision de l'Approche globale des migrations ${ }^{64}$. Toutefois, aussi longtemps que les migrations climatiques constitueront une faible priorité en matière de gouvernance, il ne faut pas trop attendre de ce processus de consultation ${ }^{65}$.

L'analyse que nous avons menée sur les instruments internationaux régionaux relatifs aux réfugiés et leur applicabilité aux migrants climatiques a permis de montrer que certains de ces instruments ne sont pas applicables du tout, et que l'applicabilité de certains autres demeure incertaine. Par conséquent, les migrants climatiques ne peuvent pas s'appuyer sur un cadre juridique clair. La contribution concrète du droit international des réfugiés à la protection des migrants climatiques est donc, bien que non négligeable, peu significative. Les victimes de catastrophes soudaines, nécessitant une protection essentiellement temporaire, se trouvent dans une position légèrement plus confortable sur le plan légal par rapport aux victimes de dégradations environnementales lentes qui ont souvent besoin de protection permanente. En outre, la première catégorie de victimes peut souvent compter sur la bonne volonté (temporaire) de ses voisins et d'autres États.

Dans une certaine mesure, il existe pour les autorités nationales, qui souhaitent accorder une protection aux migrants climatiques, un espace juridique offrant des instruments dont l'applicabilité n'est pas automatique mais possible. En réalité, le problème résulte aussi bien d'un manque de volonté politique de protéger les migrants climatiques que de l'absence de cadre juridique. En ce qui concerne l'absence de cadre juridique efficace, l'adoption d'une

S113/08, Bruxelles, 14 mars 2008. Voir aussi Commission Européenne (CE), Communication on strengthening the global approach to migration: increasing coordination, coherence and synergies, COM/2008/0611 final, 8 october 2008, disponible sur : http://eurlex.europa.eu/LexUriServ/LexUriServ.do?uri=COM:2008:0611:FIN:EN:HTML.

63. Conseil européen, "The Stockholm Programme - An Open and Secure Europe Serving and Protecting Citizens”, Brussels, 16 octobre 2009, Official Journal C 115/1-38 of 4 May 2010 ; Kraler A., Cernei T., et Noack M., "'Climate refugees': Legal and policy responses to environmentally induced migration ", Étude demandée par la commission des libertés civiles, de la justice et des affaires intérieures du Parlement européen, Direction générale des politiques internes, Département thématique C - Droits des Citoyens et Affaires Constitutionnelles, 2011.

64. Commission Européenne, Communication from the Commission to the European Parliament, the Council, the European Economic and Social Committee and the Committee of the Regions on "Delivering an area of freedom, security and justice for Europe's citizens: Action Plan Implementing the Stockholm Programme”, COM(2010) 171 final, Bruxelles, 20 april 2010, non publié au Journal Officiel, disponible sur : http://eurlex.europa.eu/LexUriServ/LexUriServ.do?uri=COM:2010:0171:FIN:EN:PDF.

65. Somerville W., op. cit. 
nouvelle loi accordant aux migrants climatiques forcés le droit d'entrer et de séjourner dans un pays étranger pose plusieurs difficultés ${ }^{66}$. Tout d'abord, la faisabilité politique d'une telle loi dans le futur (proche) est incertaine. La politique d'immigration est l'une des rares expressions fortes de la souveraineté nationale : la possibilité de décider de l'admission des étrangers sur le territoire national relève en conséquence des États. Deuxièmement, l'adoption d'une nouvelle convention au niveau international résulte d'un long processus, alors que le problème de la migration climatique exige une réponse urgente. Troisièmement, la définition des ayant droit est délicate : déterminer dans la loi qui doit être inclus dans la catégorie des migrants climatiques et qui doit en être exclu, et donc qui est admissible dans un pays étranger et qui ne l'est pas. Il est en effet malaisé d'établir des distinctions claires entre la migration volontaire/migration forcée, les conditions environnementales/les mauvaises conditions socio-économiques et la pauvreté. Enfin, l'adoption d'un instrument international semblable à celui de l'asile devra être complétée par le développement de programmes internationaux de réinstallation des migrants partant d'un pays (en développement) vers un autre (développé). En l'absence de tels programmes, ce sont les pays en développement qui risqueraient d'accueillir sur leurs territoires la grande majorité des migrants climatiques : d'une part, la migration climatique est susceptible de se produire principalement dans certaines régions (la pauvreté empêchant de nombreuses personnes de traverser les continents) et, d'autre part, les effets du changement environnemental global touchent plus particulièrement les pays en développement.

L'analyse ci-dessus a porté sur des mouvements forcés transfrontaliers de personnes et sur le droit international des réfugiés. Les mouvements internes de personnes sont, quant à eux, régis par le droit pour les déplacés internes. Les Principes directeurs relatifs au déplacement interne ${ }^{67}$ sont un ensemble de principes développés en 1998 par Francis M. Deng, ex-Représentant spécial du Secrétaire général des Nations unies pour les personnes déplacées dans leur propre pays, et par la suite adoptés par les Nations unies. Ils sont principalement basés sur le droit international des droits de l'Homme et le droit international humanitaire. Les Principes directeurs guident les États concernant la protection des personnes déplacées internes contre, pendant et après un déplacement. Les premiers principes - concernant la protection contre le déplacement - indiquent que les Principes directeurs jouent un rôle dans la prévention des migrations climatiques.

Les Principes directeurs s'appliquent à toutes les personnes obligées de se déplacer au sein des frontières nationales, indépendamment de la cause $\mathrm{du}$ départ (principe 2) et donc aussi aux migrants climatiques internes. Puisqu'il

66. La libre circulation des personnes au sein de l’Union européenne constitue une exception à la règle.

67. Les Principes directeurs relatifs au déplacement interne, E/CN.4/1998/53/Add.2. 
est probable que la majorité des personnes affectées négativement par les changements environnementaux globaux ne disposent pas des moyens financiers ou autres nécessaires pour se déplacer à l'étranger, et étant donné la réticence des États à admettre des étrangers sur leurs territoires, le déplacement forcé au sein des pays est susceptible d'augmenter considérablement en cette ère du changement climatique. Par conséquent, le droit du déplacement interne est particulièrement pertinent et important. Cependant, les Principes directeurs étant juridiquement non contraignants, on peut se demander s'ils seront effectivement appliqués par les États. Plusieurs États ont intégré ces principes ou des versions adaptées dans leur législation et politiques nationales ${ }^{68}$. C'est le cas d'une vingtaine d'États répartis sur différents continents. Au niveau sous-régional, la Région des Grands Lacs en Afrique a adopté, en 2006, le Protocole sur la protection et l'assistance aux personnes déplacées internes ${ }^{69}$, dans le cadre du Pacte de 2006 sur la sécurité, la stabilité et le développement dans la région des Grands Lacs ${ }^{70}$. Sur le plan régional, l'Union Africaine a adopté en octobre 2009 la Convention pour la protection et l'assistance des personnes déplacées en Afrique (Convention de Kampala) ${ }^{71}$. Cette Convention souligne plus spécifiquement les catastrophes d'origine naturelle ou humaine, y compris le changement climatique, comme l'une des principales causes du déplacement interne ${ }^{72}$. Elle accorde une protection identique aux migrants climatiques et aux autres déplacés internes ${ }^{73}$.

Les instruments régionaux et sous-régionaux, mentionnés ci-dessus, s'appuient sur les Principes Directeurs relatifs au déplacement interne tant pour leur contenu, que pour la définition de «personnes déplacées internes ». Le droit du déplacement interne est donc en expansion progressive grâce à la mise en ouvre nationale et régionale des Principes directeurs. La notion de « personnes déplacées internes » prend toute son importance car elle comprend toutes les personnes forcées de quitter leurs foyers après la survenue d'une catastrophe, quelle que soit la nature de la catastrophe. Il n’est donc pas nécessaire de déterminer si une catastrophe particulière a été causée par le changement climatique ${ }^{74}$. L'efficacité du droit du déplacement interne dans le

68. Voir le Projet Brookings-Bern sur le déplacement interne : www.brookings.edu/projects/idp/Laws-and-Policies/idp_policies_index.aspx.

69. Pour le texte du Protocole sur la protection et l'assistance aux personnes déplacées à l'intérieur de leur pays, voir : www.brookings.edu/ /media/Files/Projects/IDP/Laws\%20and\%20Policies/Great\%20Lak es/GreatLakes_IDPprotocol_2006.pdf.

70. Pour le texte du Pacte de Sécurité, de Stabilité et de Développement dans la Région des Grands Lacs, voir www.brookings.edu/ /media/Files/Projects/IDP/Laws\%20and\%20Policies/Great\%20Lak es/GreatLakes_Pact2006.pdf.

71. Le texte de la Convention peut être consulté sur le site de l’Union africaine : www.africaunion.org.

72. Article 1(k), Article 4(4)(f), Article 5(4) et Article 12(3) de la Convention de Kampala.

73. Maru M.T., "The Kampala Convention and its Contribution in Filling the Protection Gap in International Law”, Journal of Internal Displacement, 1, 2011, pp. 61-130.

74. Kälin W., "Conceptualising Climate-Induced Displacement”, in McAdam J. (ed.), Climate 
contexte de la protection des migrants climatiques dépend de la volonté politique et de la capacité des États à appliquer réellement les droits des personnes déplacées internes et à exercer leurs responsabilités énoncées dans les instruments susmentionnés.

\section{Le droit international des migrations}

Comme évoqué dans l'introduction, le terme "migration ", dans le présent article, comprend le déplacement interne ou externe, volontaire ou forcé de personnes. Le droit international des réfugiés peut donc être considéré comme une branche du droit international des migrations qui porte sur certains types de migrations forcées, en particulier lorsque le droit à la vie est menacé (par exemple, en cas de persécution ou de conflit armé). Les migrants ne relevant pas du droit des réfugiés, ceux qui ont quitté leur lieu de résidence habituelle involontairement en raison d'une menace pour leur vie, ou ceux qui ont quitté leur lieu de résidence habituelle dans le seul but d'améliorer leur qualité de vie, relèvent du droit international des migrations. On compte parmi eux les «migrants économiques » qui souhaitent améliorer leur niveau de vie, pour lesquels le degré de contrainte dans leur décision de se déplacer peut varier considérablement ${ }^{75}$. Selon les spécificités de chaque cas, les migrants climatiques appartiennent à l'une ou l'autre de ces catégories.

Les « travailleurs migrants » constituent une composante importante du phénomène migratoire dans le monde. Ils quittent leur lieu de résidence habituelle pour se déplacer dans un autre pays ou dans le but d'exercer un emploi 76 . Certains migrants climatiques correspondent à ce type de migration. Alors qu'aujourd'hui le pouvoir de décider de l'admission des migrants au sein du marché du travail national demeure principalement au niveau national ${ }^{77}$, il est particulièrement intéressant d'examiner les droits que les travailleurs migrants peuvent exercer dans le pays d'emploi sur la base du droit international des migrations. L'instrument le plus important et le plus pertinent est la Convention internationale sur la protection des droits de tous les travailleurs migrants et des membres de leur famille (Convention sur les travailleurs migrants), adoptée par l'Organisation des Nations unies en 1990 78. La

Change and Displacement. Multidisciplinary Perspectives, Hart Publishing, Oxford, 2010, pp. 81-103.

75. Sur les questions de terminologie, voir Organisation internationale pour les migrations (OIM), World Migration Report 2008: Managing Labour Mobility in an Evolving Global Economy, Genève, IOM, 2008, pp. 491-501 qui donne un aperçu des termes et des définitions en matière de migrations.

76. Organisation internationale pour les migrations (OIM), World Migration Report 2008: Managing Labour Mobility in an Evolving Global Economy, Geneva, IOM, 2008, p. 495.

77. Voir note 64.

78. 18 décembre 1990, 2220 UNTS 93. La Convention sur les travailleurs migrants est entrée en vigueur le 1 er juillet 2003. D’autres instruments pertinents sont les diverses conventions du Bureau International du Travail (OIT) sur les travailleurs migrants. Certaines de ces conventions sont largement ratifiées par les États tandis que d'autres ne le sont pas (Weissbrodt D., 
Convention sur les travailleurs migrants définit un travailleur migrant à l'article 2 (1) comme « une personne qui va exercer, exerce ou a exercé une activité rémunérée dans un État dont elle n'est pas ressortissante ». Cette définition inclut tous les migrants quel que soit leur statut juridique : migrants en situation régulière, migrants sans-papiers, victimes de la traite qui sont souvent exploités et mal rémunérés pour leur travail, etc. Toutefois, afin de décourager la migration irrégulière, la Convention sur les travailleurs migrants accorde plus de droits aux migrants en situation régulière qu'aux sans-papiers ${ }^{79}$. Basée sur les instruments internationaux des droits de l'Homme, la Convention sur les travailleurs migrants ne fait que réaffirmer les normes existantes relatives aux droits humains sans en élaborer de nouvelles. Néanmoins, peu d'États ont ratifié cette Convention, et ceux qui l'ont fait sont principalement des États qui sont des pays d'origine et non de destination ${ }^{80}$. Il y a tout simplement un manque de volonté politique pour protéger les travailleurs migrants.

La Convention sur les travailleurs migrants, ainsi que d'autres instruments juridiques internationaux sur les migrations (du travail), sont dans la pratique pertinents uniquement pour les migrants climatiques qui parviennent à s’engager dans une activité salariée dans un État étranger. Pour revenir à la question de l'admission des migrants sur un territoire étranger, la stratégie consistant à faciliter la migration de travail, permanente ou temporaire (circulaire), par des accords bilatéraux ou multilatéraux entre les gouvernements, est une approche intéressante dans le cadre du problème des migrations climatiques ${ }^{81}$. Au lieu de considérer la migration comme un dernier recours de survie, la migration peut être considérée comme une stratégie positive d'adaptation aux changements environnementaux. Les programmes de migration $\mathrm{du}$ travail, en particulier ceux à caractère circulaire, peuvent contribuer de manière substantielle à la reconstruction de zones et à la reconstitution de communautés sinistrées par le biais de versements réalisés par les travailleurs migrants ${ }^{82}$. En outre, ces programmes de migration de main-d'œuvre peuvent jouer un rôle important dans l'adaptation de l'environnement des communautés locales. Pour les pays développés qui sont confrontés à un vieillissement de la population et à un manque de main d'œuvre, les forces de travail étrangères (en particulier temporaires) sont les bienvenues. Cette situation gagnantgagnant-gagnant (pour le pays d'origine, pour le pays d'accueil et pour la main-d'œuvre migrante) rend cette stratégie, centrée sur l'adaptation, plus faisable politiquement qu'une stratégie basée sur de nouveaux droits que pour-

The human rights of non-citizens, Oxford, Oxford University Press, 2008, pp. 183-185).

79. Weissbrodt D., op. cit., pp. 182-183 et 185-186.

80. Weissbrodt D., ibid., pp. 186-188.

81. De Moor N., "Temporary Labour Migration for Victims of Natural Disasters: The Colombia-Spain Model", in Leighton M., Shen X. et Warner K. (eds.), Climate Change and Migration: Rethinking Policies for Adaptation and Disaster Risk Reduction, 15/2011, Publication Series of UNU-EHS, April 2011, p. 92-103.

82. Organisation Internationale pour les Migrations (OIM), Migration, Climate Change and Environmental Degradation, Genève, 2009, p. 8. 
rait définir une nouvelle Convention en matière d'asile. La facilitation ou l'organisation de la migration du travail peut donc apporter une réelle contribution à l'atténuation des difficultés rencontrées par les migrants climatiques. Il s'agit en fait de soutenir la migration volontaire en vue de réduire la migration forcée ${ }^{83}$.

Cet article a analysé le rôle de plusieurs branches pertinentes du droit international en ce qui concerne les migrations climatiques. Le droit international de l'environnement, tout en accordant une attention limitée à la migration climatique, joue implicitement un rôle important dans la prévention de cette migration à travers la protection de l'environnement naturel. Le droit international des droits humains, même s'il nécessite une application plus rigoureuse, remplit une fonction normative et préventive importante dans la protection des migrants climatiques. Le droit international des réfugiés est palliatif, en protégeant les migrants forcés dont les droits ont été violés. Au niveau régional il est possible de trouver un espace juridique permettant d'inclure les migrants du climat dans le cadre de la protection des réfugiés, $\mathrm{du}$ moins concernant la migration forcée due à des changements environnementaux lents nécessitant une protection à long terme. Cependant, la volonté politique pour le réaliser est, à l'heure actuelle, généralement absente. Or, le droit des déplacés internes est particulièrement pertinent pour les déplacés climatiques, pour autant sa mise en œuvre doit être renforcée. Enfin, la facilitation de la migration du travail, en augmentant les possibilités de migrations légales du travail, peut apporter une contribution significative à l'atténuation du problème de la migration climatique. Le développement de programmes de migration (circulaire) de main-d'œuvre, sur la base d'accords bilatéraux ou multilatéraux entre les gouvernements, devrait donc être encouragé. Le droit international des migrations du travail, tel qu'il existe actuellement, est particulièrement pertinent en ce qui concerne les droits que les migrants climatiques, devenus travailleurs migrants dans un pays étranger, peuvent exercer dans le pays d'emploi. Toutefois, le dispositif en question est caractérisé par de faibles niveaux de ratification.

L'introduction de la migration climatique dans le régime international sur le changement climatique est une première étape importante dans le processus vers un cadre juridique prenant en compte les migrations climatiques. Pourtant, la décision d'accorder ou non l'asile aux personnes déplacées suite à

83. Tacoli C., "Crisis or adaptation? Migration and climate change in a context of high mobility", paper prepared for the Expert Group Meeting on Population Dynamics and Climate Change, UNFPA and IIED, 24-25 june 2009, http://www.unfpa.org/webdav/site/global/users/schensul/public/CCPD/papers/Tacoli\%20Paper.pdf ; Newland K., "Circular migration and human development”, Human Development Research Paper, 2009/42, New York, UNDP, 2009 ; Barnett J. et Webber M., “Accommodating migration to promote adaptation to climate change", document de politique publique préparé pour le Secrétariat de la Commission suédoise sur le changement climatique et le développement et le rapport sur le développement dans le monde de la Banque mondiale, équipe 2010, mars 2009, http://www.ccdcommission.org/Filer/documents/Accommodating\%20Migration.pdf. 
des changements climatiques, ou de faciliter la migration de travail en tant que stratégie d'adaptation, reste une question de souveraineté nationale, et doit être traitée par la législation et les politiques nationales et régionales sur les migrations et non pas par le droit international de l'environnement.

Les quatre branches du droit international ont donc un rôle spécifique et important dans le traitement des migrations climatiques en liaison avec le ratio legis de chacune de ces branches. En pratique, néanmoins, plusieurs lacunes existent, liées souvent au processus de mise en œuvre et à la volonté politique. Des efforts au niveau international devraient être déployés pour renforcer les mécanismes existants et développer de nouveaux systèmes efficaces au sein de chaque branche.

En raison des fonctions spécifiques propres à chaque branche du droit international, chacune séparément ne peut pas être suffisamment efficace pour traiter de manière appropriée le problème des migrations forcées liées aux changements environnementaux globaux. Leurs contributions respectives sont complémentaires et, par conséquent, les quatre branches du droit doivent être appliquées simultanément et de manière coordonnée. En effet, une solution globale au problème des migrations climatiques implique à la fois différents types de mécanismes de prévention et différentes réponses. La prévention doit être au cœur d'une stratégie mondiale devenue urgente (mieux vaut prévenir que guérir) et les cadres appropriés pour cela sont le droit international de l'environnement d'une part et le droit international des droits de l'Homme (y compris le droit sur les déplacés internes) d'autre part. Dans la mesure où la migration est perçue comme une forme d'adaptation au changement environnemental global, le droit des migrations consiste en un autre moyen pour prévenir les migrations forcées. Pour ceux qui n'ont pas d'autre choix que de fuir et de traverser les frontières en raison de problèmes environnementaux, une protection internationale est nécessaire. Le cadre approprié pour faire face à ces situations est le droit international des réfugiés. Enfin, le renforcement des droits de l'Homme est pertinent pour les personnes qui quittent leur lieu de résidence à la recherche de meilleures conditions de vie ailleurs. Dans cette hypothèse, les cadres juridiques les plus appropriés sont le droit international des droits de l'Homme (y compris le droit sur les déplacés internes) et le droit international des migrations (du travail).

Le droit de l'environnement, le droit relatif aux droits de l'Homme, le droit des réfugiés et le droit de la migration, constituent les quatre pièces du puzzle des migrations climatiques. Séparés ils ont une fonction limitée, mais pris ensemble, ils forment un ensemble satisfaisant. Les juristes, les organisations spécialisées et les décideurs devraient en conséquence penser et travailler d'une manière interdisciplinaire afin de parvenir à une solution cohérente au problème des migrations climatiques. 